\title{
Access to secondary restorative dental care in rural Scotland
}

Referral for secondary restorative dental care in rural and urban areas of Scotland: Findings from the Highlands \&t Islands Teledentistry Project N. M. Nuttall, M. S. Steed and M. A. Donachie Br Dent J 2002; 192: 224-228

\section{Objectives}

To compare the reported level of use of secondary care services for restorative dental care in rural and urban areas of Scotland.

\section{Design}

Postal questionnaire survey

\section{Subjects and Methods}

Postal questionnaire sent to all dentists in the Highland region, the island regions in Scotland and Dumfries \& Galloway $(\mathrm{n}=150)$ and an equal number were sampled from the remainder of Scotland stratified by health board area. Non-respondents were sent 2 reminders after which $62 \%$ of the sample had responded.

\section{Results}

Most dentists (85\%) who practised in what they considered were urban areas of Scotland said they felt that they had good access to a secondary referral service. Whereas most of those who practised in what they considered were rural areas either said they had no access to such a service (26\%) or that access was difficult (53\%), only $3 \%$ of those in urban areas said they had no access to a secondary restorative consultative service compared with $14 \%$ of dentists practising in rural areas of mainland Scotland and 54\% of those practising on Scottish islands.

\section{Conclusions}

The survey suggests the people of the Scottish islands and some of the remoter parts of the Scottish mainland would be among those who might benefit from improvement in access to a restorative dentistry consultant service.

\section{IN BRIEF}

- Access to specialist restorative dental care is affected by remoteness from urban centres and is a particular problem in the Island regions of the country.

- Most dentists in urban areas said they felt that they had good access to a secondary referral service whereas most of those in rural areas either said they had no access to such a service or that access was difficult.

- The most commonly referred conditions in the opinion of general dental practitioners were; assessment and treatment planning for tooth wear and referral for apicectomy.

\section{COMMENT}

An increasing concern in the NHS is to improve both the speed and access to medical and dental care in the UK. A significant challenge is to provide this care to those in the population that live in rural areas and a particular difficulty is delivery of specialist advice and support to primary care providers working in more isolated communities. One might expect that in a speciality like restorative dentistry, where expert advice to general dental practitioners might be 'thin on the ground' in places, the challenge to providing local access to all will be significant.

A well documented method of meeting this type of challenge is through the development of a telemedicine approach, linking centres of expertise to local (usually rural) primary care surgeries. More recently such approaches have been applied in dentistry by the development of teledentistry videoconferencing based systems to provide diagnostic and treatment planning expertise at a distance. However before investing in such an approach it is wise to identify and quantify the problem. This initial report from the Highlands and Islands Teledentistry Project is an attempt to do this.

In Scotland, where much of the population is remote from a centre of expertise and where some patients, due to social deprivation, find even relatively short distances to centres daunting, there would appear to be a potential barrier for many to seeking advice from a dental consultant. This is against a background of a possible increasing need for restorative expertise. Therefore, this study seeks to determine whether dental practioners working in remote or rural areas believe that they have problems of access, for their patients, to secondary restorative advice and care. The authors suggest that, based on such data, decisions may then be made on whether providing such a service (presumably via teledentistry) might be worth consideration.

In this study 150 questionnaires were sent to all of the dentists in the Highlands and Islands in Scotland and an equal number were sent to a sample of practitioners from the remainder of Scotland. In spite of a follow-up strategy to non-respondents the response rate was a little low at $62 \%$ but the authors put forward valid reasons why this is unlikely to cause a significant bias in the results.

The results indicate that whilst most dentists practising in urban areas in Scotland felt they had good access to a secondary restorative referral service, the majority of those practicing in rural areas either had no access (26\%) or it was difficult (53\%). A more stark contrast was in the finding that whilst only $3 \%$ of urban dentists stated that they had no access to a restorative consultative service, the results for dentists in rural areas was $14 \%$ and $54 \%$ for those practising in the Scottish islands.

The conclusion is that there is a substantial proportion of the Scottish population who would benefit from improved access to such a service. Bearing in mind the title of the project that this paper is reporting it seems likely that the authors are now pursuing a teledentistry solution. It will be interesting to see in future reports how they progress in applying such a solution.

Professor Malcolm Jones

Dean of the Dental School, UWCM, Cardiff, Wales. 